\title{
Modelling the role of slips and twins in magnesium alloys under cyclic shear
}

\author{
H. Wang ${ }^{1,2, *}$, P.D. Wu ${ }^{1}$, J. Wang ${ }^{2}$ \\ ${ }^{1}$ Department of Mechanical Engineering, McMaster University, Hamilton, Ontario, L8S 4L7, \\ Canada \\ ${ }^{2}$ Materials Science and Technology Division, Los Alamos National Laboratory, Los Alamos, \\ NM, 87545, USA
}

\begin{abstract}
Magnesium alloys under cyclic shear show different deformation behaviour from that under cyclic tension-compression, for example, symmetry vs. asymmetry of stress-strain curves. Using the Twinning-DeTwinning (TDT) model, we studied the role of different deformation mechanisms, slip systems, twinning and detwinning, in shear deformation behaviour of magnesium alloys. The results indicate that the nearly symmetrical shear stress-shear strain curve unlike the asymmetrical stress-strain curve under cyclic tension-compression is ascribed to the dominated prismatic slips and the simultaneous activation of deformation twinning and detwinning under reverse shear. The typical shear texture observed in experiments is ascribed to the activation of extension twin that induces the extension strain along normal direction of the shear plane under simple shear.
\end{abstract}

Keywords: Twinning; Detwinning; Cyclic shear; Magnesium alloys; Crystal plasticity.

\footnotetext{
* Corresponding authors. Dr. Huamiao Wang, Tel. +1-505-664-0321; E-mail: wanghm@lanl.gov.
} 


\section{Introduction}

Magnesium ( $\mathrm{Mg}$ ) alloys are the lightest structural materials but have poor formability at room temperature because of insufficient available deformation mechanisms. Modelling deformation behaviour of $\mathrm{Mg}$ alloys under different loading conditions will provide insights into fabricating structural components. Under complex strain paths, such as cyclic tensioncompression and strain path changes, detwinning is activated and induces the characteristic phenomena: unsymmetrical hysteresis loops of stress strain curves [1-7], inelasticity during unloading [8-10], and the low yield stress upon load reversals [11,12], etc. Employing the Twinning-DeTwinning (TDT) models [13-15], these characters have been correctly captured and understood in terms of the activity of twinning and detwinning.

Magnesium alloys under cyclic shear show different deformation behaviour from that under cyclic tension-compression, for example, symmetry vs. asymmetry of stress-strain curves [7, 16-17]. Lou et al. [2] and Zhang et al. [18] conducted cyclic shear and torsion of magnesium alloys and found that the unsymmetrical hysteresis loops of the stress strain curves during cyclic tension-compression were not apparent under cyclic shear/torsion even at large deformation. Zhang et al. [19] experimentally observed that simple shear could improve the ductility of magnesium alloys and ascribed this to the activity of deformation twinning. In addition, twinning also results in a plastic deformation perpendicular to the shear plane during shear or torsion of Mg alloys [2, 20-28]. Zhang et al. [19] also observed that the basal pole in the (0002) pole figure tends to rotate $45^{\circ}$ away from the RD after simple shearing along the RD. They ascribed this to the high activity of prismatic slip systems as suggested by Kang et al. [29]. According to these experimental observations, the difference in deformation behaviour of $\mathrm{Mg}$ alloys between cyclic shear and compression-tension loadings has been qualitatively ascribed to the change in the 


\section{Deformation mechanisms under shear deformation}

Magnesium alloy sheets exhibit strong basal texture with $c$-axis nearly perpendicular to the sheet plane while $a$-axis randomly distributed in the sheet plane (Figure 1). Each grain is subjected to pure shear, i.e., the non-zero stress components of each grain are $\sigma_{12}=\sigma_{21}=\tau$ (where $\tau$ is the applied shear stress; 1 refers to rolling direction (RD), 2 refers to transverse direction (TD), and 3 refers to normal direction (ND)).

The activity of different deformation mechanisms can be predicted according to Schmidt factor analysis. For a grain oriented as shown in Figure 1a, the three basal slip systems are described as the slip direction $\boldsymbol{s}^{\alpha}=(\cos \phi, \sin \phi, 0)$ with $\phi=\theta, \theta+60^{\circ}, \theta+120^{\circ}$ and the same normal direction $\boldsymbol{n}^{\alpha}=(0,0,1)$. Therefore basal slip systems will not be activated because the resolved shear stress $\tau^{\alpha}=\boldsymbol{s}^{\alpha} \cdot \boldsymbol{\sigma} \cdot \boldsymbol{n}^{\alpha}$ is equal to zero. For the three prismatic slip systems, $\boldsymbol{s}^{\alpha}=(\cos \phi, \sin \phi, 0)$ and $\boldsymbol{n}^{\alpha}=(-\sin \phi, \cos \phi, 0)$, the resolved shear stress is equal to 
$\tau^{\alpha}=\tau \cos 2 \phi$ with respect to $\phi=\theta, \theta+60^{\circ}, \theta+120^{\circ}$. The pyramidal slip systems are rarely activated and thus are not considered here because of its low mobility. For six extension twins, $\boldsymbol{s}^{\alpha}=\left(\frac{\sqrt{3} a}{\rho} \sin \phi,-\frac{\sqrt{3} a}{\rho} \cos \phi, \frac{c}{\rho}\right)$ and $\boldsymbol{n}^{\alpha}=\left(-\frac{c}{\rho} \sin \phi, \frac{c}{\rho} \cos \phi, \frac{\sqrt{3} a}{\rho}\right)$ with respect to $\phi=\theta, \theta+60^{\circ}, \theta$ $+120^{\circ}, \quad \theta+180^{\circ}, \quad \theta+240^{\circ}, \quad \theta+300^{\circ}$, the resolved shear stress is equal to $\tau^{\alpha}=\frac{\sqrt{3} a}{\rho} \tau \sin 2 \phi \approx \frac{1}{2} \tau \sin 2 \phi$, where $\rho=\sqrt{3 a^{2}+c^{2}}$. The factor $\tau^{\alpha} / \tau$ is plotted in Fig. $1 \mathrm{~b}$ as a function of $2 \theta$. Unlike unidirectional twinning in $\mathrm{Mg}$, gliding dislocations can be activated regardless of the sign of $\tau^{\alpha} / \tau$, the absolute value of $\tau^{\alpha} / \tau$ is plotted in Figure $1 \mathrm{~b}$ for prismatic slip systems. The shadows indicate the ranges of $\tau^{\alpha} / \tau$ under pure shear. The range of the factor $\tau^{\alpha} / \tau$ associated with prismatic slip systems with highest possibility of activation is from 0.866 to 1 , while that for extension twins is from 0.25 to 0.5 and -0.5 to -0.25 . During cyclic shear, shear stress reversal does not change the activity of prismatic slip systems but changes the activity of twinning. Twinning only takes place in a grain where the resolved shear stress is positive and detwinning only takes place in a twinned grain where the resolved shear stress is negative. Therefore the activity of extension twin will be nearly doubled upon shear stress reversal. Of course, their relative activities among prismatic slip systems, twinning, and detwinning depend on their critical resolved shear stresses (CRSSs).

\section{TDT model}

TDT model $[13,15]$ has been developed to deal with twinning and detwinning deformation mechanisms according to deformation physics including twin nucleation, twin growth, twin shrinkage and re-twinning that retain the glide of twinning dislocations (TDs). TDs including their characteristics and mobility have been studied and characterized at atomic scale by using high resolution transmission electron microscopy and molecular dynamics simulations 
[30-35]. Under a loading that enables twinning, twin nucleation $(\mathrm{TN})$ creates a fresh twin in a grain and the grain is then split into an untwined domain (matrix) and a twinned domain (twin). The nucleated twin grows according to eqs. (1) and (2) under the resolved shear stress associated with TDs. With respect to the resolved shear stress computed in the matrix and in the twin, the migration of twin boundaries results matrix reduction (MR) and twin propagation (TP), respectively. Detwinning can be accomplished through two mechanisms, twin shrinkage and retwinning. Twin shrinkage is the reverse mechanism of twin growth and re-twinning (RT) corresponds to twin nucleation in a twin. Again, with respect to the resolved shear stress computed in the matrix and in the twin, twin shrinkage includes matrix propagation (MP) and twin reduction (TR), respectively. TDT model has been implemented into the elastic viscoplastic self-consistent (EVPSC) model [36], named as EVPSC-TDT that has been successfully employeed in modelling plastic deformation of magnesium alloys under different loadings $[13,22,23,36-42]$. Associated with each operation, the shear rates are expressed as:

$$
\begin{aligned}
& \dot{\gamma}_{I}^{\alpha}=\left\{\begin{array}{cc}
\dot{\gamma}_{0}\left|\tau^{\alpha} / \tau_{c r}^{\alpha}\right|^{1 / m} & \tau^{\alpha}>0 \\
0 & \tau^{\alpha} \leq 0
\end{array} \quad \text { if } I=T N, T P, M P, R T\right. \\
& \dot{\gamma}_{I}^{\alpha}=\left\{\begin{array}{cc}
-\dot{\gamma}_{0}\left|\tau^{\alpha} / \tau_{c r}^{\alpha}\right|^{1 / m} & \tau^{\alpha}<0 \\
0 & \tau^{\alpha} \geq 0
\end{array} \quad \text { if } I=M R, T R\right.
\end{aligned}
$$

where $\dot{\gamma}_{0}$ and $m$ are constants of the reference shear rate and the rate sensitivity, and $\tau^{\alpha}$ and $\tau_{c r}^{\alpha}$ are the resolved shear stress and the threshold shear stress, respectively. The evolution of the threshold shear stress for each slip or twinning system is described by the extended Vocehardening law as

$$
\hat{\tau}^{\alpha}=\tau_{0}^{\alpha}+\left(\tau_{1}^{\alpha}+\theta_{1}^{\alpha} \Gamma\right)\left(1-\exp \left(-\theta_{0}^{\alpha} \Gamma / \tau_{1}^{\alpha}\right)\right)
$$


Here $\tau_{0}^{\alpha}, \tau_{1}^{\alpha}, \theta_{0}^{\alpha}$ and $\theta_{1}^{\alpha}$ are the associated hardening parameters, and $\Gamma=\sum_{\alpha} \int \dot{\gamma}^{\alpha} d t$ is the accumulated shear strain.

The twin volume fractions associated with twin nucleation and re-twinning are small, the net evolution of the twin volume fraction $f^{\alpha}$ is mainly due to twin growth (MR and TP) and twin shrinkage (MP and TR):

$$
\left.\dot{f}^{\alpha}=\left(1-\sum_{\beta} f^{\beta}\right)\left(\left|\dot{\gamma}_{M R}^{\alpha}\right|-\left|\dot{\gamma}_{M P}^{\alpha}\right|\right) / \gamma^{t w}+f^{\alpha}\left(\left|\dot{\gamma}_{T P}^{\alpha}\right|-\mid \dot{\gamma}_{T R}^{\alpha}\right)\right) / \gamma^{t w}
$$

where $\gamma^{t w}$ is the characteristic twinning shear strain and is 0.129 for extension twinning systems of magnesium alloys. In order to control the twinning and detwinning process, the threshold twin volume fraction $V^{\text {th }}=\min \left(1.0, A_{1}+A_{2} V^{\text {eff }} / V^{a c c}\right)$ is defined to terminate twinning, where $A_{1}$ and $A_{2}$ are two material constants, $V^{\text {eff }}$ and $V^{a c c}$ are the weighted volume fraction of the twinned region and volume fraction of twin terminated grains. $A_{1}$ and $A_{2}$ are obtained through numerical fitting the standard experiments, e.g., tension and compression [13].

\section{Results and discussions}

The room temperature elastic constants, the reference slip/twinning rate and the rate sensitivity are taken from Wang et al. [13]. The initial crystallographic texture of the sheet [2] is discretized to 4104 grains with independent orientations and weights. The discretized initial texture is used for all the simulations in this work. Hardening parameters (Table 1) are adjusted through fitting both the monotonic uniaxial tension and compression tests with the initial texture from the same set of experiments [13]. The initial CRSS $\tau_{0}^{\text {Pri }}$ associated with prismatic slip systems is less than $2 \tau_{0}^{E T}$ of extension twin, which indicates prismatic slip systems are initially more active than extension twin (Fig. 1b). According to the planar simple shear test performed 
by Lou et al. [2], the boundary condition for modelling the planar simple shear with the TDT model is: $\dot{\varepsilon}_{12}=\dot{\gamma} / 2=0.002 s^{-1}\left(-0.002 s^{-1}\right.$ for reverse shear $), \quad \dot{\varepsilon}_{13}=\dot{\varepsilon}_{23}=\dot{\varepsilon}_{22}=0$ and $\sigma_{11}=\sigma_{33}=0$

The AZ31B sheet is sheared in the sheet plane to a shear strain of $18 \%\left(\mathrm{P}^{0} \rightarrow \mathrm{P}^{1}\right)$, and then reversely sheared to $4 \%\left(\mathrm{P}^{1} \rightarrow \mathrm{P}^{2}\right)$ and again reversely sheared to $20 \%\left(\mathrm{P}^{2} \rightarrow \mathrm{P}^{3}\right)$. Fig. 2 shows the shear strain shear stress curves of AZ31B sheet under cyclic shear. The nearly symmetrical shear stress shear strain curves of the cyclic loop from $\mathrm{P}^{1}$ to $\mathrm{P}^{2}$ and $\mathrm{P}^{2}$ to $\mathrm{P}^{3}$ are observed in both simulation and experiment. Fig. 3 shows the predicted relative activities of various deformation mechanisms. The relative activity of a deformation mechanism is defined as the percentage of the absolute shear rate contributed by the deformation mechanism. During the stage of $\mathrm{P}^{0} \rightarrow \mathrm{P}^{1}$, the most active deformation mechanism is associated with prismatic slip systems, while extension twin and basal slip systems are active too. The activation of basal slip systems during simulations is ascribed to the non-zero resolved shear stress in the grains that are not perfectly orientated with c-axis perpendicular to the sheet plane. Upon shear stress reversal from $\mathrm{P}^{1}$ to $\mathrm{P}^{2}$, extension twin becomes the most active and is almost doubled compared to the shear from $\mathrm{P}^{0}$ to $\mathrm{P}^{1}$. The relative activity of twinning (MR and TP) and detwinning (MP and TR) is shown in Fig. 4. Only twinning is active from $\mathrm{P}^{0}$ to $\mathrm{P}^{1}$, while twinning and detwinning are both active in the processes from $\mathrm{P}^{1}$ to $\mathrm{P}^{2}$ and from $\mathrm{P}^{2}$ to $\mathrm{P}^{3}$. The evolution of the twin volume fraction in Fig. 5 shows that the twin volume fraction increases monotonically from $\mathrm{P}^{0}$ to $\mathrm{P}^{1}$ (the predicted twin volume fraction at $\gamma=0.1$ agrees well with the measured one. The measured one taken from Lou et al. [2] is indicated by a circle in Figure 5), decreases and increases sequentially from $\mathrm{P}^{1}$ to $\mathrm{P}^{2}$ and from $\mathrm{P}^{2}$ to $\mathrm{P}^{3}$. The shear stress reversal upon more pre-twinned volume fraction detwins more at the beginning of shear process. The nearly equivalent activities of extension twin 
between the processes of $\mathrm{P}^{1}$ to $\mathrm{P}^{2}$ and $\mathrm{P}^{2}$ to $\mathrm{P}^{3}$ result in the approximate symmetry of shear stress shear strain curve.

Figure 6 shows strains associated with each deformation mechanism as a function of the applied shear strain. The applied shear strain $\gamma$ is decomposed into elastic shear strain $\gamma^{e}$ and plastic shear strain $\gamma^{p}$. The plastic strain $\gamma^{p}$ is further decomposed into strains associated with slip $\left(\gamma^{\text {slip }}\right)$ and twinning $\left(\gamma^{\text {twin }}\right)$. The strain analysis shows that (1) the total shear strain is mostly accommodated by plastic deformation; (2) the plastic shear strain is mainly contributed from slip; (3) plastic shear deformation variation with respect to $\mathrm{P}^{1}$ upon shear stress reversal is accommodated by both slip and twinning that are comparable. Interestingly, (4) the twin shear strain accommodation from $\mathrm{P}^{1}$ to $\mathrm{P}^{2}$ is nearly symmetrical to that from $\mathrm{P}^{2}$ to $\mathrm{P}^{3}$. This nearly symmetrical behavior is attributed to the simultaneous occurrence of both twinning and detwinning (TP, TR, MP and MR) as shown in Fig. 5. Finally, a through thickness extension strain $\varepsilon_{33}$ corresponds to deformation twinning and agrees well with the experiments [2]. This observation is consistent with other experiments [20,21,23,24].

The predicted texture evolution of AZ31B sheets under cyclic shear is presented in Fig. 7 in terms of the pole figures. The typical shear texture $\left(\mathrm{P}^{1}\right.$ in Fig. 7$)$ that was observed in experiments $[19,21,43,44]$ was successfully reproduced using the EVPSC-TDT model that includes basal, prismatic and pyramidal slip systems, twinning and detwinning deformation mechanisms. Most importantly, the quantitative modelling provides insight in understanding the appearance of two peaks with $c$-axes of the grains nearly lied in the RD-TD plane along the direction $\sim 45^{\circ}$ to RD from TD to RD. As a comparison, we re-simulated the texture of the AZ31B sheet during simple shear by excluding the contribution of all slip systems to texture 
evolution in the EVPSC-TDT. It is found that the predicted texture at $\mathrm{P}^{1}$ shows typical shear texture again, indicating that twinning is mainly responsible for the appearance of two peaks. Fig. 8 schematically shows that the stress state of simple shear is equivalent to a biaxial normal stress state. The compression stress activates extension twin and reorients the $c$-axes of grains toward the direction of the compression stress. Therefore a typical shear texture is formed under simple shear with two peaks in the pole figures. Upon shear stress reversal, the $c$-axes of the most of the twinned grains are reoriented back to the center of the pole figures. Therefore the two peaks in the pole figures at $\mathrm{P}^{1}$ disappear at $\mathrm{P}^{2}$. However the untwinned grains from the previous shear stress (at the center of the pole figure at $\mathrm{P}^{1}$ ) are twinned by the reversed shear stress. Two peaks are formed $\left(\mathrm{P}^{2}\right)$ with $c$-axes of the grains nearly lied in the RD-TD plane along the direction $\sim 45^{\circ}$ to $\mathrm{RD}$ from $\mathrm{RD}$ to $\mathrm{TD}$. The subsequent shear reversal evolves the texture back to be similar to the one at $\mathrm{P}^{1}$ (see $\mathrm{P}^{3}$ in Fig. 7).

\section{Conclusions}

Shear deformation behaviour of $\mathrm{Mg}$ alloys under cyclic shear are studied by using the EVPSC-TDT model. The key features observed in experiments are well captured and explained as follows. (1) Prismatic slip systems prevail over basal slip systems during shear deformation, (2) the nearly symmetrical shear stress-shear strain curve is ascribed to the simultaneous activation of twinning and detwinning under reversing shear, (3) the typical shear texture observed in experiments is ascribed to the activation of extension twin, and (4) the extension strain along the normal direction of the sheet plane under simple shear is accommodated by extension twins. 


\section{Acknowledgement}

This research was supported by the Natural Sciences and Engineering Research Council of Canada (NSERC) and by the Ontario Ministry of Research and Innovation. JW was supported by the US department of Energy, Office of Basic Energy Sciences (Project No: FWP06SCPE401).

\section{References}

[1] Roberts, C.S., John Wiley \& Sons, Inc., New York (1960).

[2] Lou, X.Y., Li, M., Boger, R.K., Agnew, S.R., Wagoner, R.H., Int. J. Plasticity 23 (2007) 44-86.

[3] Wu, L., Agnew, S.R., Brown, D.W., Stoica, G.M., Clausen, B., Jain, A., Fielden, D.E., Liaw, P.K., Acta Mater. 56 (2008) 3699-3707.

[4] Wu, L., Jain, A., Brown, D.W., Stoica, G.M., Agnew, S.R., Clausen, B., Fielden, D.E., Liaw, P.K., Acta Mater. 56 (2008) 688-695.

[5] Hong, S.G., Park, S.H., Lee, C.S., J. Mater. Res. 25 (2010) 784-792.

[6] Hong, S.G., Park, S.H., Lee, C.S., Acta Mater. 58 (2010) 5873-5885.

[7] Wu, L., Agnew, S.R., Ren, Y., Brown, D.W., Clausen, B., Stoica, G.M., Wenk, H.R., Liaw, P.K., Mater. Sci. Eng.A527 (2010) 7057-7067.

[8] Gharghouri, M.A., Weatherly, G.C., Embury, J.D., Root, J., Philo. Mag. A79 (1999) 1671-1695.

[9] Cáceres, C.H., Sumitomo, T., Veidt, M., Acta Mater. 51 (2003) 6211-6218.

[10] Mann, G.E., Sumitomo, T., Cáceres, C.H., Griffiths, J.R., Mater. Sci. Eng. A456 (2007) 138-146.

[11] Kleiner, S., Uggowitzer, P.J., Proceedings of the 1st International Light Metals Technology Conference. The CRC for CAST Metals Manufacturing, Brisbane Australia, (2003) pp. 327-332.

[12] Kleiner, S., Uggowitzer, P.J., Mater. Sci. Eng. A379 (2004) 258-263.

[13] Wang, H., Wu, P.D., Tomé, C.N., Wang, J., Mater. Sci. Eng. A555 (2012) 93-98.

[14] Wang, H., Wu, P.D., Wang, J., Int. J. Plasticity 47 (2013) 49-64.

[15] Wang, H., Wu, P.D., Wang, J., Tomé, C.N., Int. J. Plasticity 49 (2013) 36-52.

[16] Li, M., Lou, X.Y., Kim, J.H., Wagoner, R.H., Int. J. Plasticity 26 (2010) 820-858.

[17] Kim, J.H., Kim, D., Lee, Y.S., Lee, M.G., Chung, K., Kim, H.Y., Wagoner, R.H., Int. J. Plasticity 50 (2013) 66-93.

[18] Zhang, H., Huang, G., Wang, L., Roven, H.J., Xu, Z., Pan, F., Scripta Mater. 69 (2013) 49-52.

[19] Zhang, J.X., Yu, Q., Jiang, Y.Y., Li, Q.Z., Int. J. Plasticity 27 (2011) 768-787.

[20] Staroselsky, A., Anand, L., Int. J. Plasticity 19 (2003) 1843-1864.

[21] Beausir, B., Toth, L.S., Qods, F., Neale, K.W., 2009. J. Eng. Mater. Technol. 131 (2009) 011108.

[22] Wang, H., Wu, Y., Wu, P.D., Neale, K.W., CMC-Computers Materials \& Continua 19 (2010) 255-284.

[23] Wu, P.D., Wang, H., Neale, K.W., Int. J. App. Mech. 4 (2012) 1250024.

[24] Guo, X.Q., Wu, W., Wu, P.D., Qiao, H., An, K., Liaw, P.K., Scripta Mater. 69 (2013) 319-322.

[25] Nave, M.D., Barnett, M.R., Scripta Mater. 51 (2004) 881-885. 
[26] Yang, P., Yu, Y., Chen, L., Mao, W., Scripta Mater. 50 (2004) 1163-1168.

[27] Choi, S.H., Shin, E.J., Seong, B.S., Acta Mater. 55 (2007) 4181-4192.

[28] Jiang, L., Jonas, J.J., Mishra, R.K., Luo, A.A., Sachdev, A.K., Godet, S., Acta Mater. 55 (2007) 3899-3910.

[29] Kang, J.Y., Bacroix, B., Brenner, R., Scripta Mater. 66 (2012) 654-657.

[30] Tomé, C.N., Beyerlein, I.J., Wang, J., McCabe, R.J., JOM 63 (2011) 19-23.

[31] Wang, J., Hirth, J.P., Tomé, C.N., Acta Mater.57 (2009) 5521-5530.

[32] Wang, J., Beyerlein, I.J., Tomé, C.N., Scripta Mater. 63 (2010) 741-746.

[33] Wang, J., Beyerlein, I.J., Hirth, J.P., Tomé, C.N., Acta Materialia 59 (2011) 3990-4001.

[34] Wang, J., Liu, L., Tomé, C.N., Mao, S.X., Gong, S.K., Mater. Res. Let. 1 (2013) 81-88.

[35] Wang, J., Yadav, S.K., Hirth, J.P., Tomé, C.N., Beyerlein, I.J., Mater. Res. Let. 1 (2013) 126-132.

[36] Wang, H., Wu, P.D., Tomé, C.N., Huang, Y., J. Mech. Phys. Solids 58 (2010) 594-612.

[37] Wang, H., Raeisinia, B., Wu, P.D., Agnew, S.R., Tomé, C.N., Int. J. Solids Struct. 47 (2010) 2905-2917.

[38] Wang, H., Wu, P.D., Gharghouri, M.A., Mater. Sci. Eng. A527 (2010) 3588-3594.

[39] Wang, H., Wu, P.D., Neale, K.W., J. Zhejiang Univ.-Sci. A11 (2010) 744-755.

[40] Wang, H., Wu, P.D., Boyle, K.P., Neale, K.W., Int. J. Solids Struct. 48 (2011) 10001010 .

[41] Wang, H., Wu, P.D., Tomé, C.N., Wang, J., Int. J. Solids Struct. 49 (2012) 2155-2167.

[42] Wang, H., Clausen, B., Tomé, C.N., Wu, P.D., Acta Mater. 61 (2013) 1179-1188.

[43] Agnew, S.R., Horton, J.A., Lillo, T.M., Brown, D.W., Scripta Mater. 50 (2004) 377-381.

[44] Agnew, S.R., Mehrotra, P., Lillo, T.M., Stoica, G.M., Liaw, P.K., Acta Mater. 53 (2005) 3135-3146. 


\section{Table and Figure Captions:}

Table 1. Material parameters used in the TDT model and determined by fitting the monotonic uniaxial tension and compression stress strain curves of AZ31B sheet.

Figure 1. (a) Schematic of magnesium alloy sheets under shear, showing orientation of grains with respect to shear; (b) The factor $\tau^{\alpha} / \tau$ as a function of $2 \theta$ with respect to prismatic slip systems (red) and twinning systems (blue).

Figure 2. The shear stress shear strain curve of magnesium alloy AZ31B sheet under simple shear.

Figure 3. The predicted relative activities of various deformation mechanisms as a function of applied shear strain for magnesium alloy AZ31B sheet under simple shear.

Figure 4. The predicted relative activities of various operations associated with extension twin as a function of applied shear strain for magnesium alloy AZ31B sheet under simple shear.

Figure 5. Evolution of twin volume fraction with applied shear strain for magnesium alloy AZ31B sheet under simple shear. The circle represents the measured twin volume fraction [2].

Figure 6. Variation of Elastic strain, plastic strain, and strains associated with slip and twin with applied shear strain. The dot represents the measured strain $\varepsilon_{33}$ [2].

Figure 7. The predicted textures at various strain levels with and without the contribution of slips to the texture

Figure 8. Equivalent stress state between simple shear and bi-axial normal stresses.

Table 1. Material parameters used in the TDT model and determined by fitting the monotonic uniaxial tension and compression stress strain curves of AZ31B sheet.

\begin{tabular}{cccccccc}
\hline Mode & $\boldsymbol{\tau}_{\mathbf{1}}(\mathrm{MPa})$ & $\boldsymbol{\tau}_{\mathbf{2}}(\mathrm{MPa})$ & $\boldsymbol{\theta}_{\mathbf{0}}(\mathrm{MPa})$ & $\boldsymbol{\theta}_{\mathbf{1}}(\mathrm{MPa})$ & $\boldsymbol{q}$ & $\boldsymbol{A}_{\mathbf{1}}$ & $\boldsymbol{A}_{\mathbf{2}}$ \\
\hline Basal & 12 & 10 & 80 & 5 & 1 & & \\
Prismatic & 80 & 35 & 400 & 20 & 1 & & \\
Pyramidal & 120 & 120 & 800 & 0 & 1 & & \\
Extension twin & 45 & 0 & 0 & 0 & 1 & 0.75 & 0.8 \\
\hline
\end{tabular}



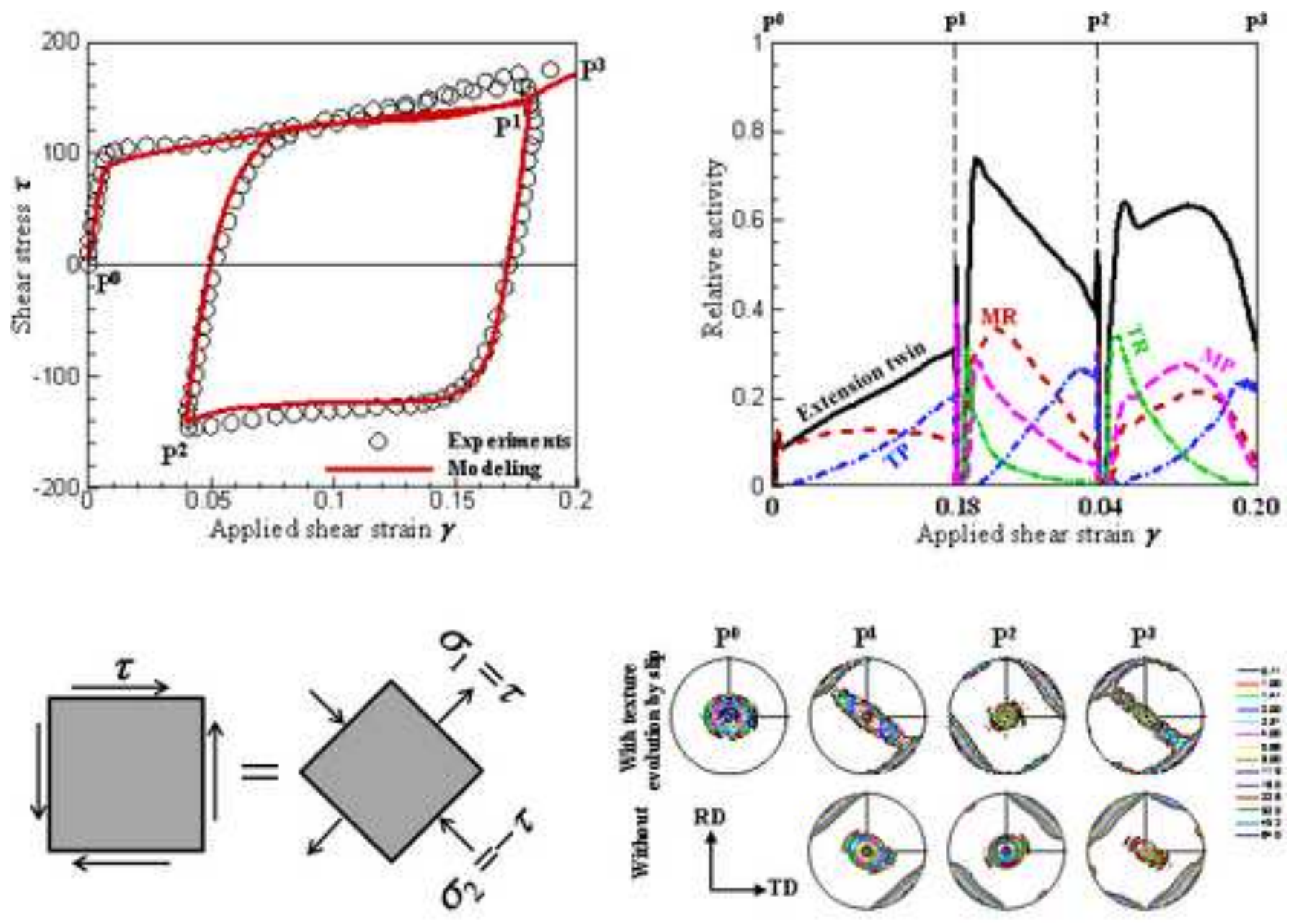

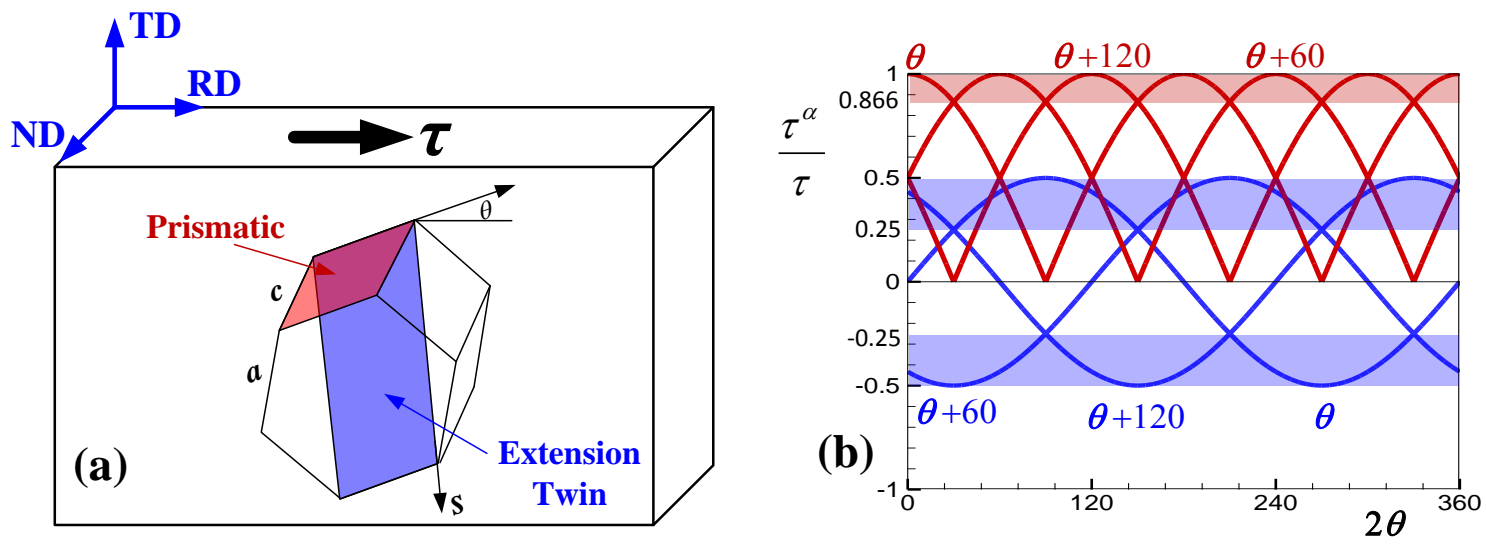

Figure 1. (a) Schematic of magnesium alloy sheets under shear, showing orientation of grains with respect to shear; (b) The factor $\tau^{\alpha} / \tau$ as a function of $2 \theta$ with respect to prismatic slip systems (red) and twinning systems (blue).

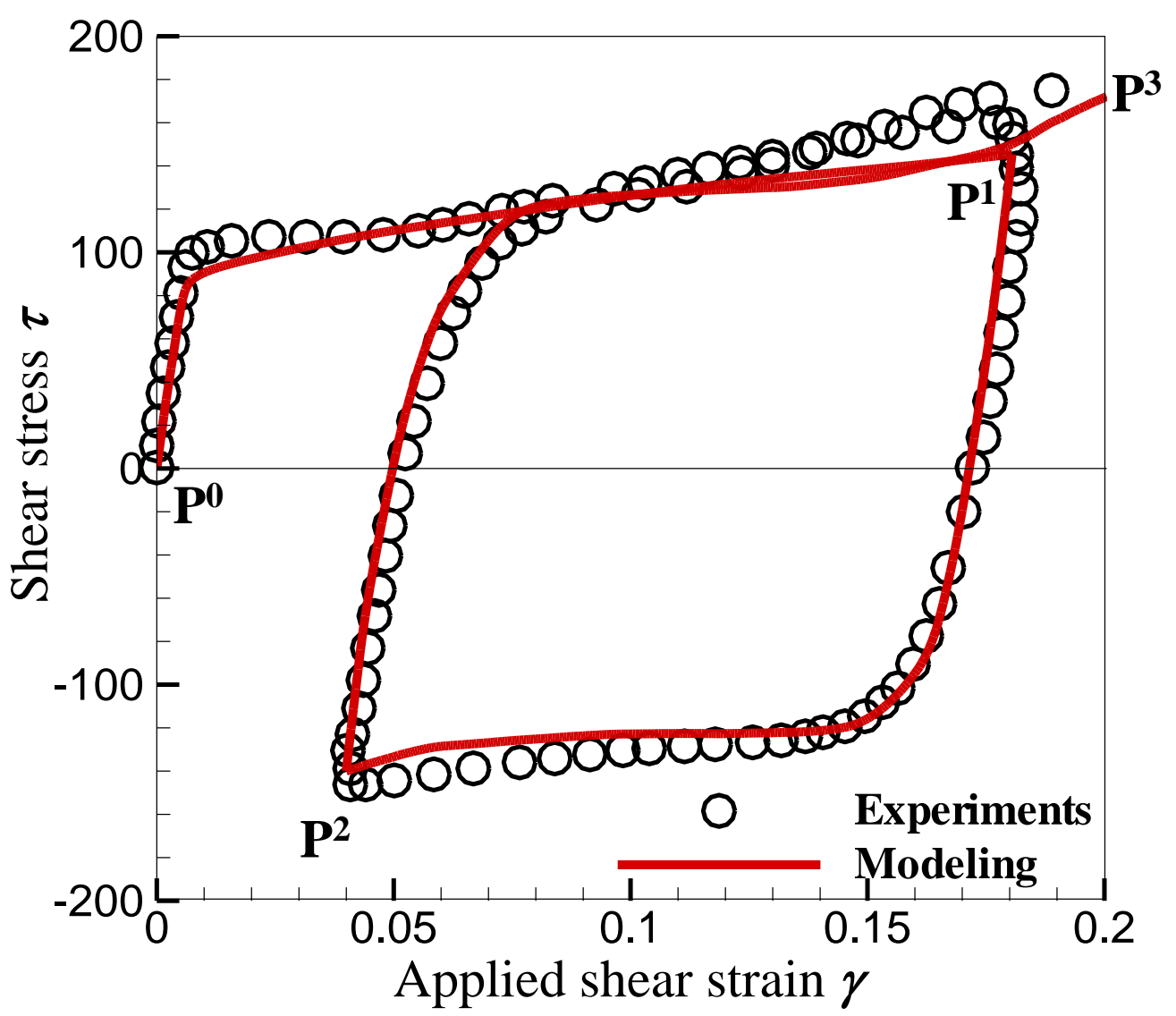

Figure 2. The shear stress shear strain curve of magnesium alloy AZ31B sheet under simple shear. 


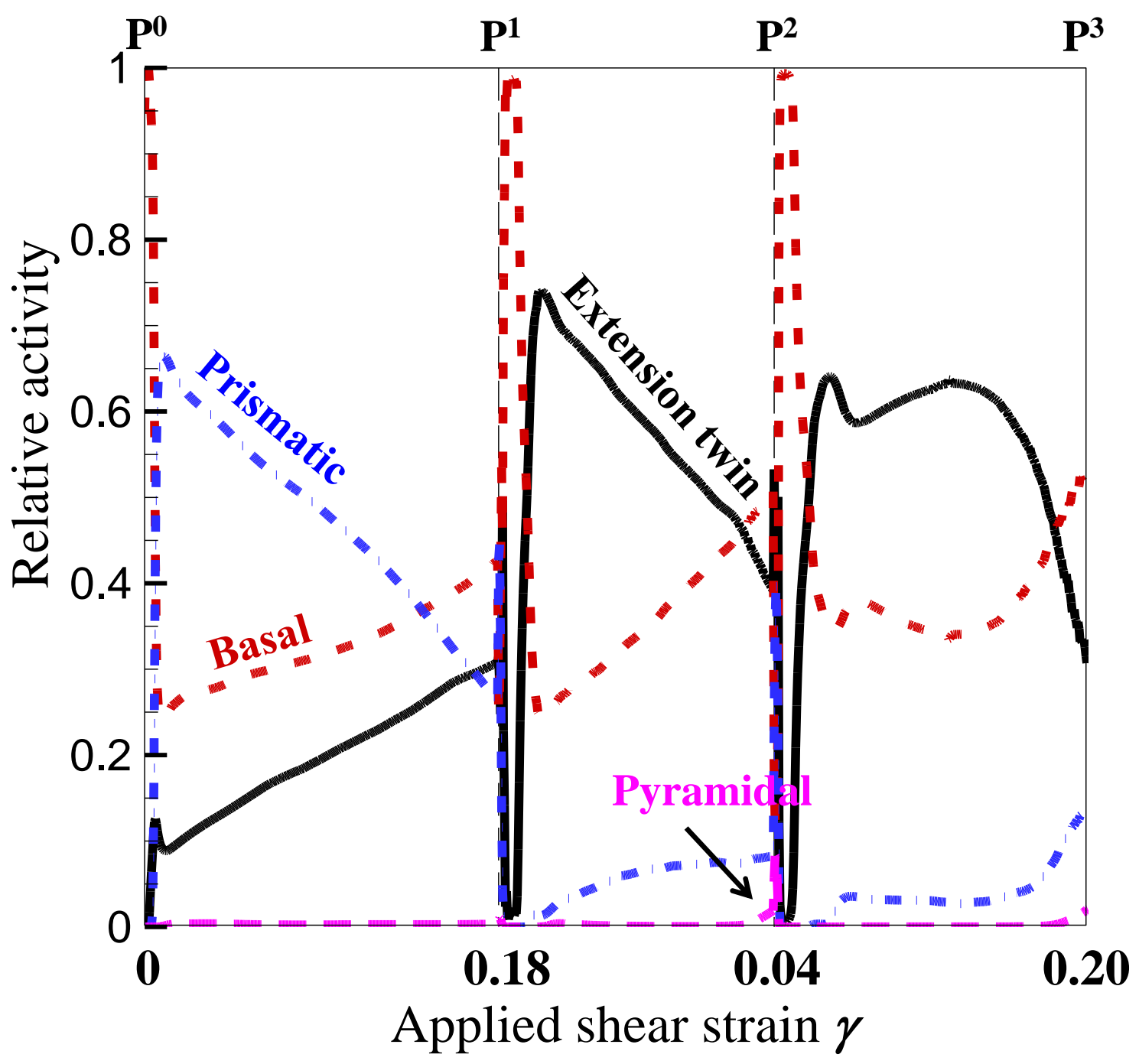

Figure 3. The predicted relative activities of various deformation mechanisms as a function of applied shear strain for magnesium alloy AZ31B sheet under simple shear. 


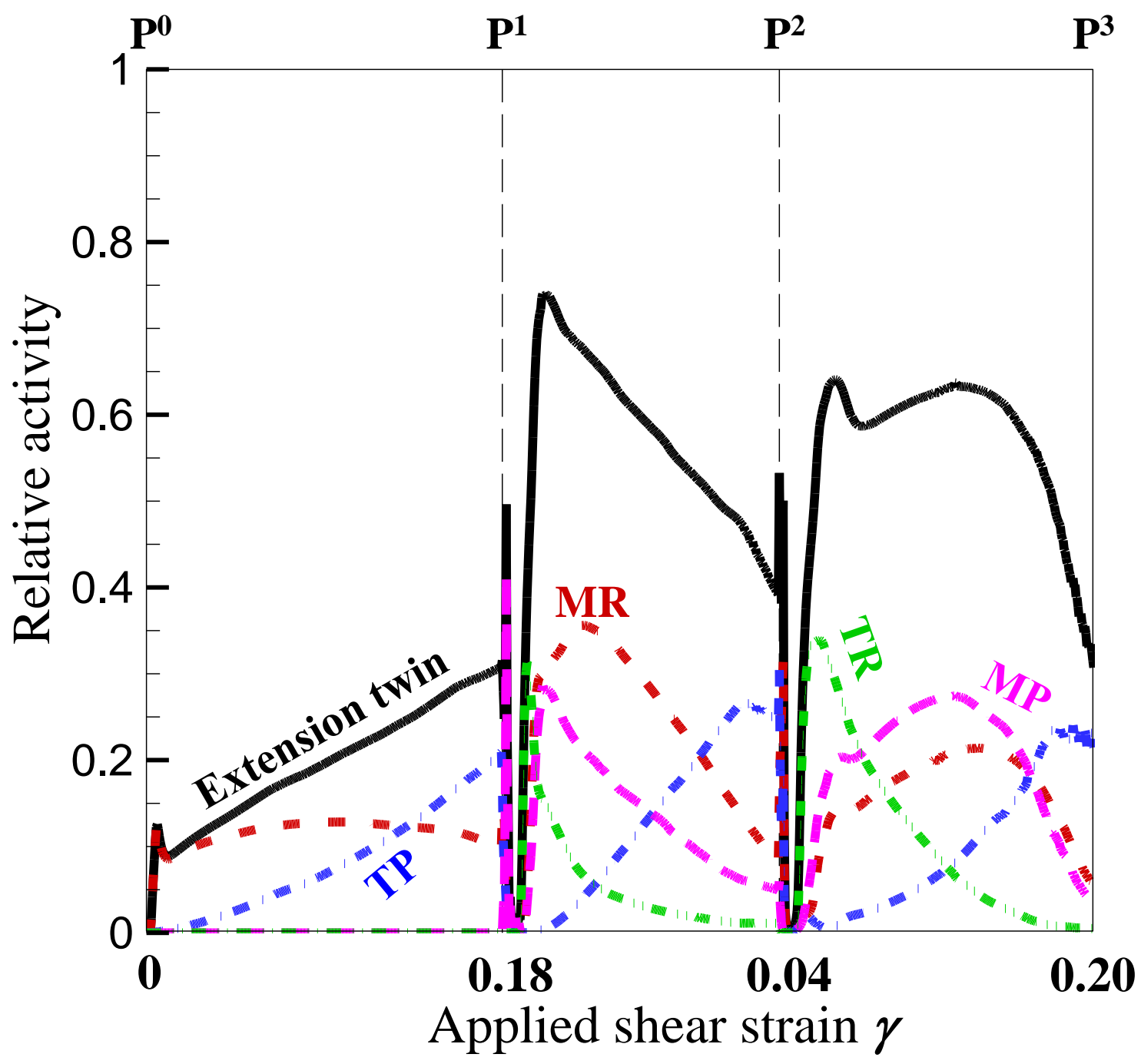

Figure 4. The predicted relative activities of various operations associated with extension twin as a function of applied shear strain for magnesium alloy AZ31B sheet under simple shear. 


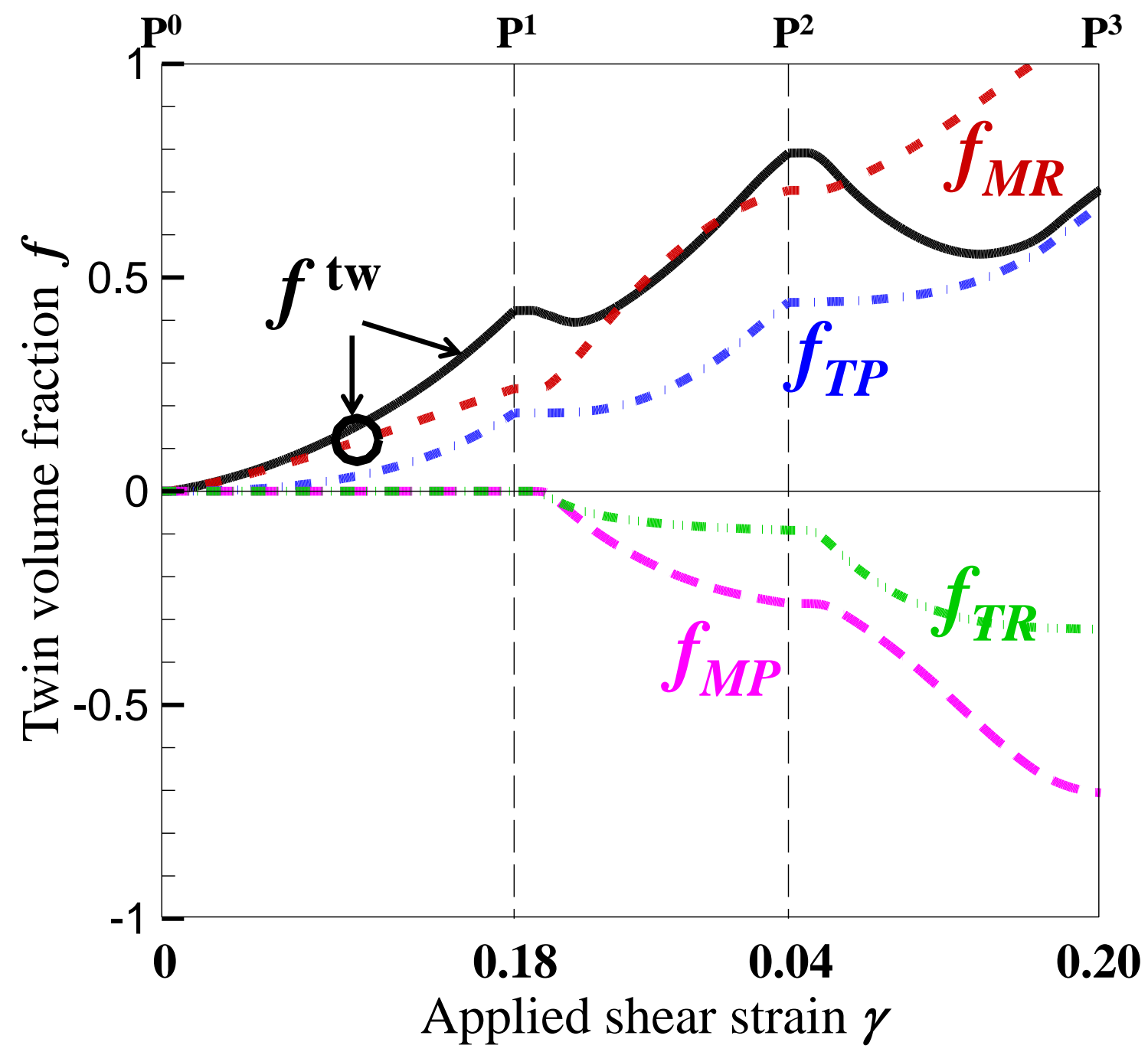

Figure 5. Evolution of twin volume fraction with applied shear strain for magnesium alloy AZ31B sheet under simple shear. The circle represents the measured twin volume fraction [2]. 


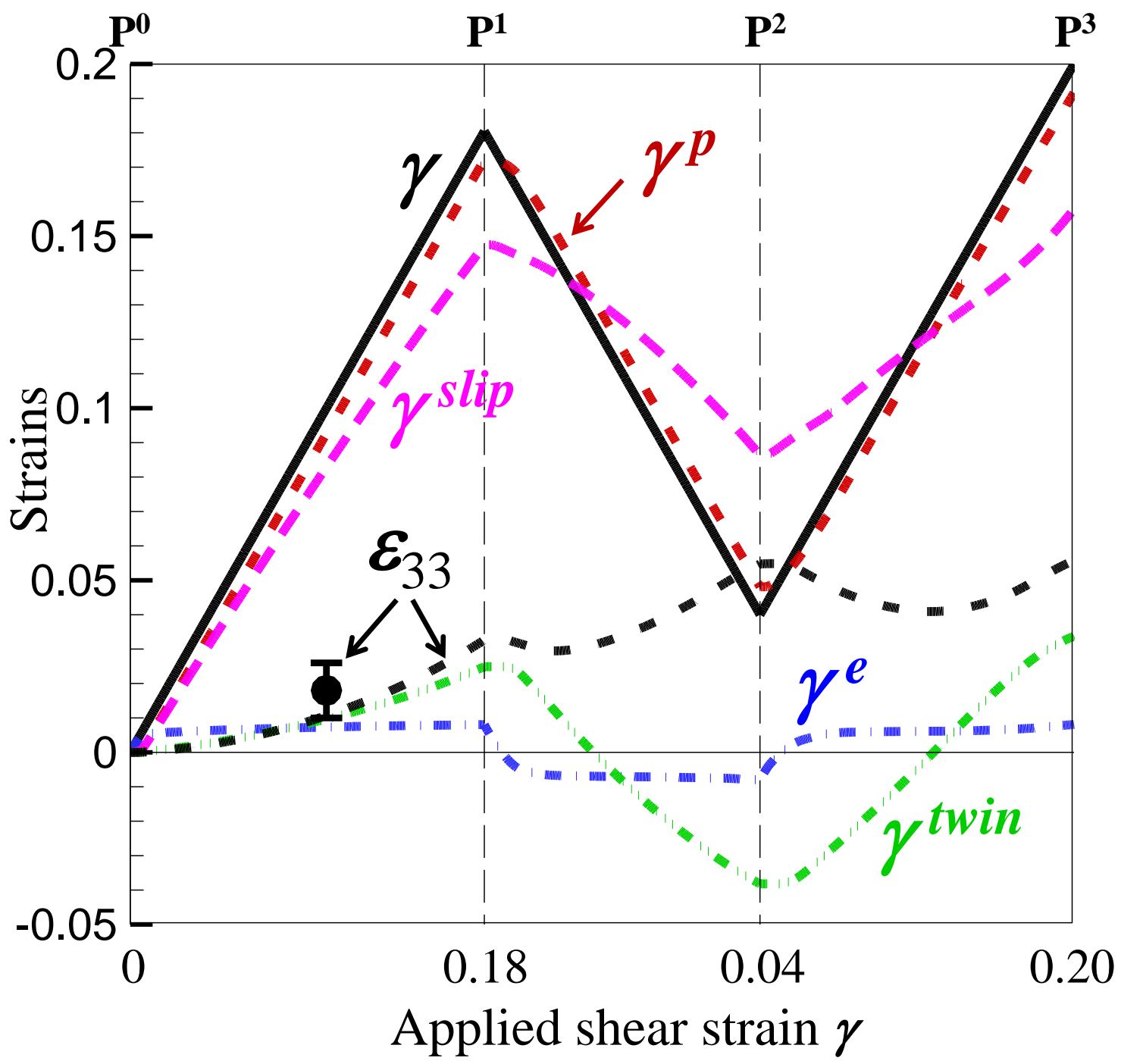

Figure 6. Variation of Elastic strain, plastic strain, and strains associated with slip and twin with applied shear strain. The dot represents the measured strain $\varepsilon_{33}$ [2]. 

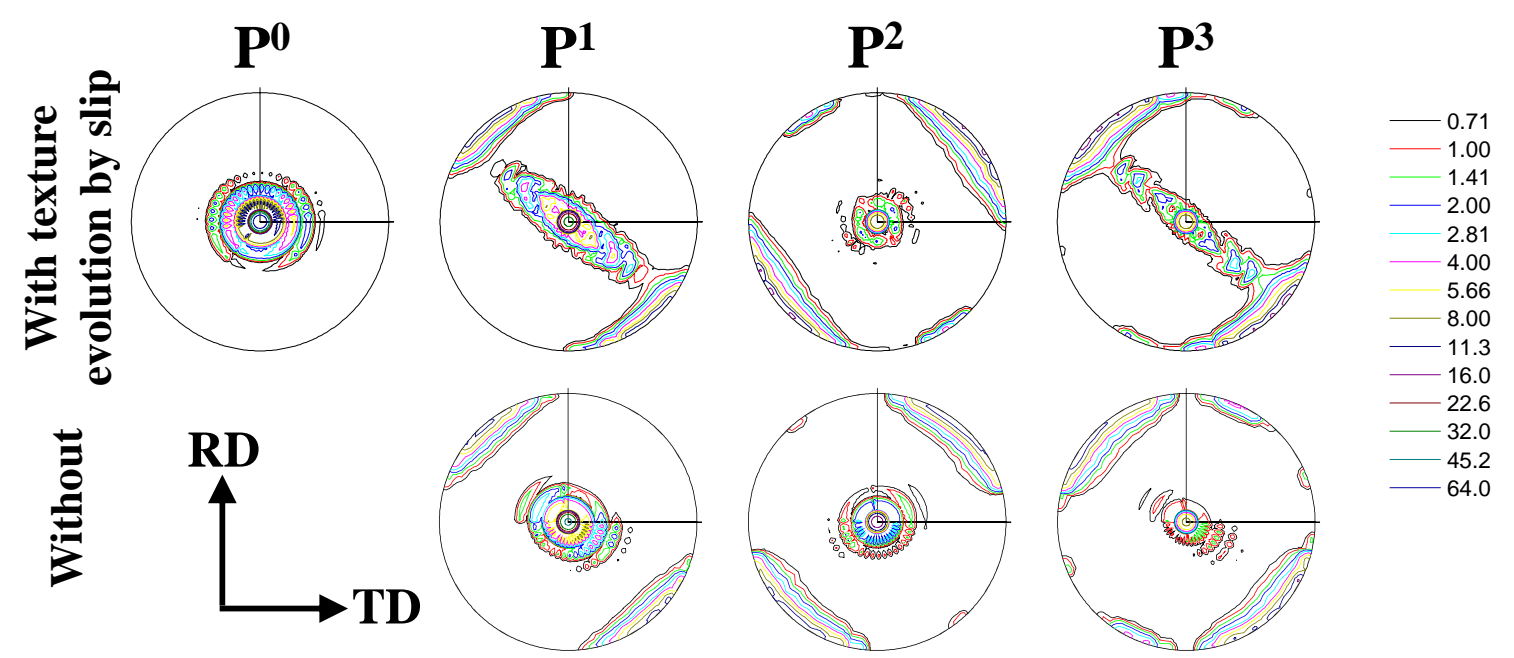

Figure 7. The predicted textures at various strain levels with and without the contribution of slips to the texture.
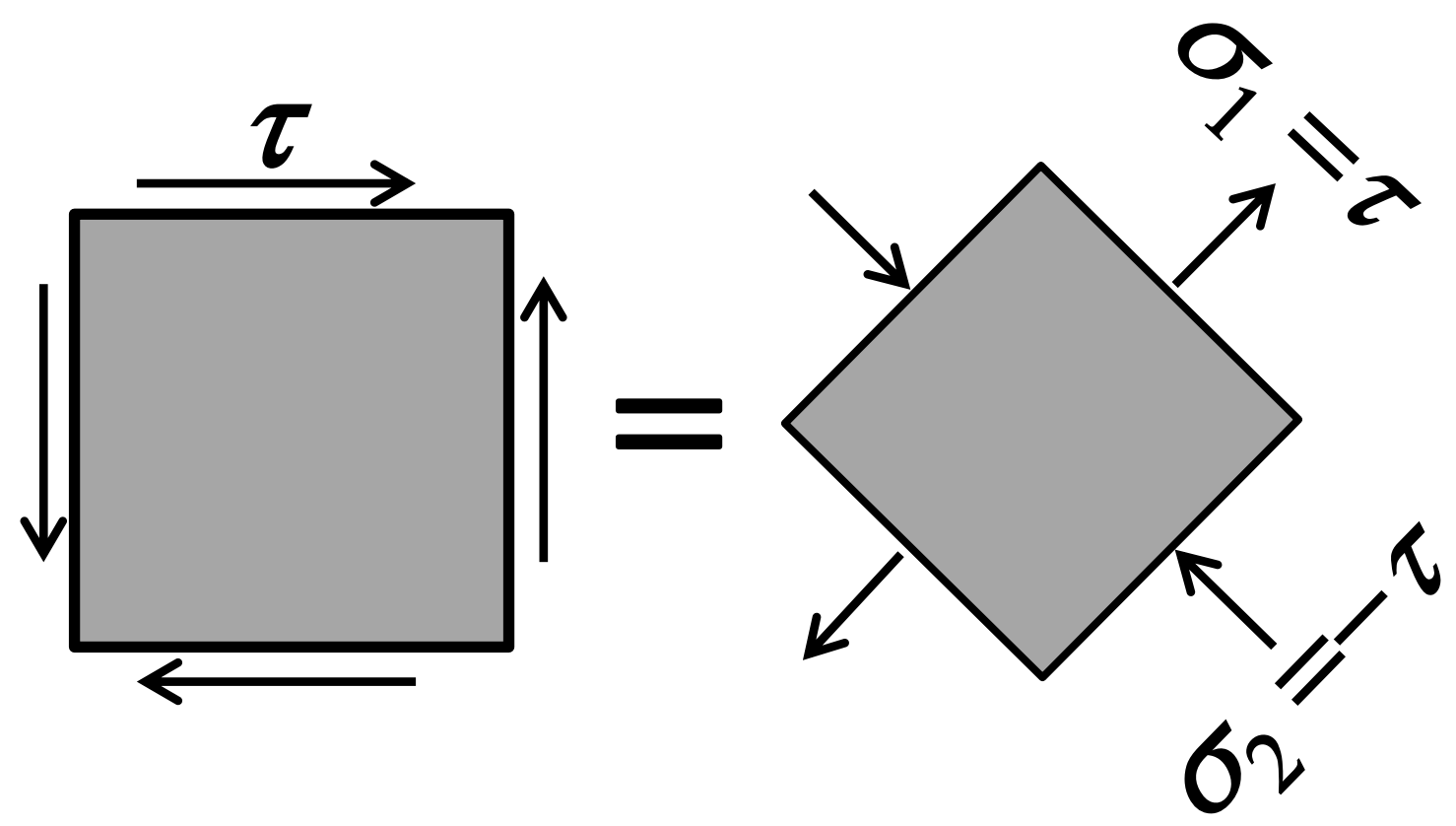

Figure 8. Equivalent stress state between simple shear and bi-axial normal stresses. 